\title{
TRUST BASED AUTOMATIC QUERY FORMULATION SEARCH ON EXPERT AND KNOWLEDGE USERS SYSTEMS
}

\author{
${ }^{1}$ Sridharan, K. and ${ }^{2}$ M. Chitra \\ ${ }^{1}$ Department of Information Technology, Panimalar Engineering College, Chennai, India \\ ${ }^{2}$ Department of Information Technology, Sona College of Technology, Salem, India
}

Received 2013-12-11; Revised 2013-12-14; Accepted 2014-02-18

\begin{abstract}
Due to enhance in complexity of services, there is a necessity for dynamic interaction models. For a serviceoriented system to work properly, we need a context-sensitive trust based search. Automatic information transfer is also deficient when unexpected query is given. However, it shows that search engines are vulnerable in answering intellectual queries and shows an unreliable outcome. The user cannot have a fulfillment with these results due to lack of trusts on blogs. In our modified trust algorithm, which process exact skill matching and retrieval of information based on proper content rank. Our contribution to this system is new modified trust algorithm with automatic formulation of meaningful query search to retrieve the exact contents from the top-ranked documents based on the expert rank and their content quality verified of their resources provided. Some semantic search engines cannot show their important performance in improving precision and lowering recall. It hence effectively reduces complexity in combining HPS and software services.
\end{abstract}

Keywords: Human Provided Services, Expert Ranking, Trust Emergence, Dynamic Trust Calculation, Metric Calculation

\section{INTRODUCTION}

The web information is enormous, unrelated and dynamic. Effort in information retrieval systems goes back many years and is well developed (Witten, 1994). The web is a vast collection of absolutely unrestrained heterogeneous documents. There is practically no control over what people can put on the web. One promising area of research is using proxy caches to build search databases. This search method is use the proxy for validated and cached information based on query given by the user. Most important search engines were designed based on conventional information retrieval methods. Federated searching reduces the time that is needed for searching several databases and also users do not need to know how to search through different interfaces (Fryer, 2004). In order to do content mining, one must first decide the problems of semantic integration across web documents.

The major problems of web mining is based on finding relevant information with low precision and unindexed information, personalizing the information with catering to personal preference in content and presentation and learning about the consumers. IE systems for the general Web are not feasible (Kosala and Blockeel, 2000).

Web services play an important role in fulfilling various sectors' objectives; web search is one of the most important among them. As the technology keeps developing and the utilization of the web services increasing, there is a large requirement of searching process to be improved to next level and faster as well. We utilize Human provided Services and system provided Services thus enabling flexible interactions in service-oriented systems.

Searching an Answer to query: There are many solutions to find the answer to a query:

- By comparing the single keyword from the query context meaning with the content one by one and extracting the answer

- By manually searching for the answer for the query from the given file is done

Corresponding Author: Sridharan, K., Department of Information Technology, Panimalar Engineering College, Chennai, India 
These solutions are not user friendly it is insufficient for the seeker to actually get to a required and right answer i.e., it lacks precisions or accuracy in providing the exact document required. Different Reputation bootstrapping for trust establishment among web services was studied (Malik and Bouguettaya, 2009).

Here in 'Automatic Query Formulation with Context Exploration and Recapture' a new method of query context identification which will help the seeker to save time by formulating query automatically for a given query to form new meaningful query based on the most possible answer and it submit into the algorithm to receive the appropriate answer by forming most possible response sentences from a cluster of answers in their respective domain database in a prioritized order with more precision or accuracy. In our further sessions we shell explain in detail how the 'Automatic Query Formulation with Context Exploration and Recapture' works.

The rest of this paper is structured as follows: In section 2 there is discussion of the related work in the expert discovery, delegation model and domain for information extraction. In Section 3 proposed system architecture and the responsibility of each component with algorithm and its functions are explained. In the section 4 we will present the results and discussion to validate our methodology, also we summarize some of the key results in the fields and in section 5 we conclude the paper.

\section{RELATED WORKS}

Information Retrieval is the routine retrieval of all pertinent documents while at the same time recovering as a small number of of the non pertinent as possible (Rijsbergen, 1979). Information drawing out aims to mine relevant information from the documents while Information recovery aims to select appropriate documents (Pazienza, 1997). The Web offers confront for Web data mining appropriate to the following characteristics of the Web (Liu and Chang, 2004): Data of every types be present on the Web. Information on the web is heterogeneous. Multiple Web pages may present the identical or similar information using completely different formats or syntaxes, which makes combination of information a challenging task, the Web is noisy that is web page typically surround a mixture of many kinds of information, e.g., main content, advertisements, routing panels, patent notices. For a particular request only fraction of the information is useful and the rest are noises and the Web is dynamic.

Jiang and Li (2010) bring about a Web log file truth preprocessing algorithm based on joint filtering. It can make customer session recognition fast and flexibly even though statistical data are not sufficient and user history visiting records are absence.

Extracting useful patterns and rules using data mining techniques in order to appreciate the users navigational behavior, so that decisions concerning site improvement or modification can then be made by humans (Ratnakumar, 2005).

Every day more business processes are opting for an open web based platform and web services for providing their services. We utilize Human provided Services thus enabling flexible interactions in service-oriented systems. There are two ways to search for solutions:

- We can manually discover an expert by asking public for their opinion and manually deciding who is responsible

- We can discover an expert from a pool of experts in an expert focal point

However, these options are not updated regularly since they don't take into account the learning curve of an expert. Thus we provide a way to dynamically rate experts according to metrics values and new trust algorithm.

We learned about the Qualitative trust modeling in SOA by Kovac and Trcek (2009).

They recommended computing a set of PageRank vectors, subjective using a set of representative topics, to capture more precisely the notion of significance with respect to a exacting topic (Haveliwala, 2002).

We got the some important study about the characterizing the influence of domain expertise on Web search behavior (White et al., 2009).

\subsection{Expertise Model}

In this section, we are discovering experts based on their skills which are given by an expert seeker in the form of a personalized query refer in Fig. 2 and data flow for the same in Fig. 3. Before an expert can provide services he has to be rated as a trusted expert. When registering to be a knowledge worker, the experts trust has to be initiated. Then based on their skills they are given a hub score. For each hub an authority is decided again based on ranks. These ranks are calculated dynamically based on success and failure. A skill model is also proposed as a classification system.

\subsubsection{New Trust Emergence}

In this, we focus on social faith to support for judge the expert facts and their skills (Schall et al., 2011), (Golbeck, 2008; 2009), (Artz and Gil, 2007). Here New trust calcualtion is done. 


\subsubsection{Personalized Expert Queries}

First basic matching is performed based on the query to find the skills and then the experts are discovered based on the information (Peng, 2010).

\subsubsection{Skill Based Model}

This develop the Model based on the expert skills refer Fig. 2. We did some changes like to assign the each id for all the well know skill domain based on the priority they are given to improve the skill identification of expert here Fig. 1.

\subsection{EXPERT DISCOVERY}

Task-based proposal on the web allow users to share their proficiency (Yang et al., 2008) or users offer their expertise by serving other users in forums or response communities (Jurczyk and Agichtein, 2007), (Agichtein et al., 2008).

Before an expert can be approached for a problem we have to first discover the appropriate expert with the right skill necessary. A skill matching algorithm is required to match the skills required and the skills of an expert.

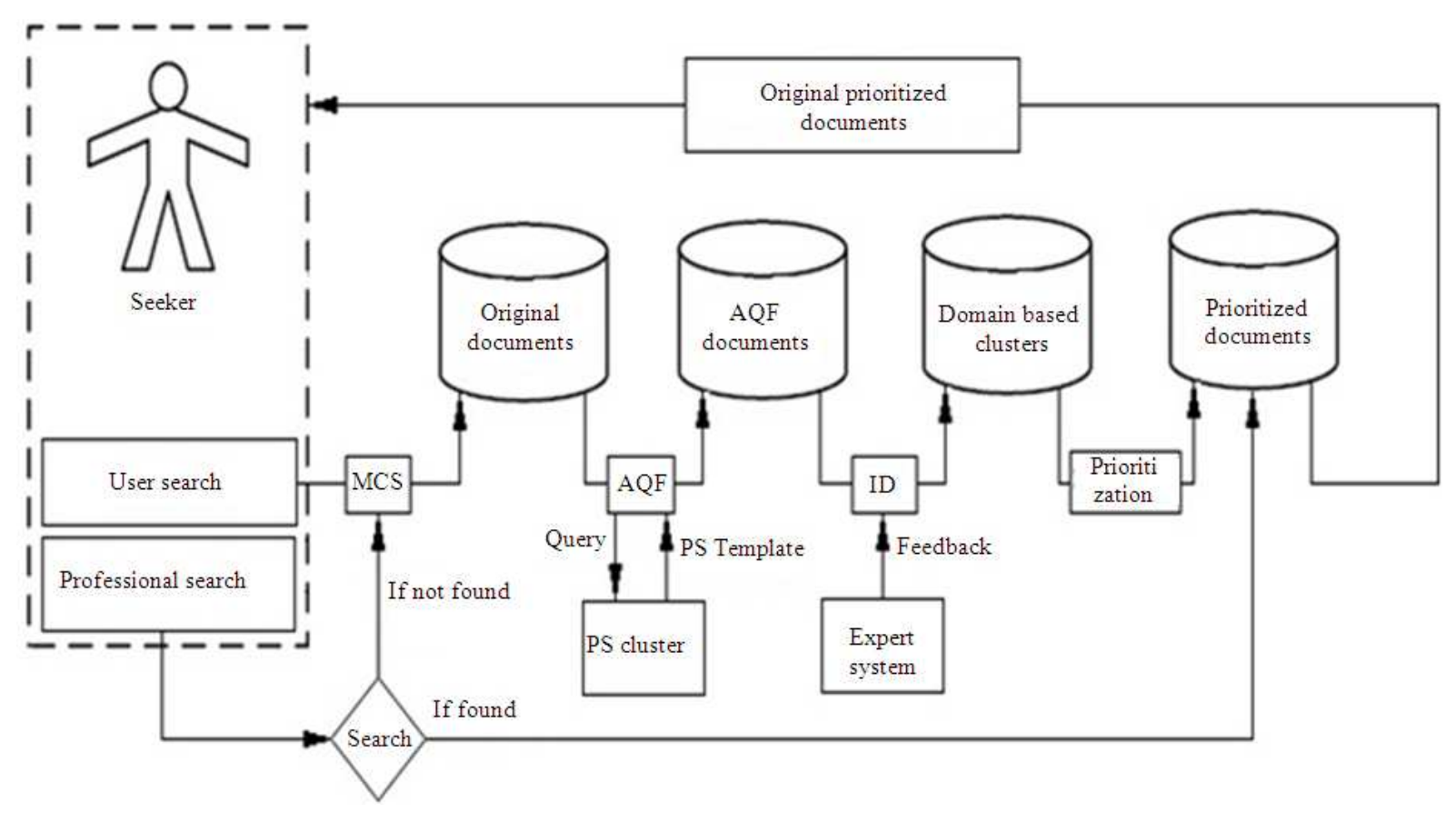

Fig. 1. Architecture for automatic query formulation with context exploration and recapture

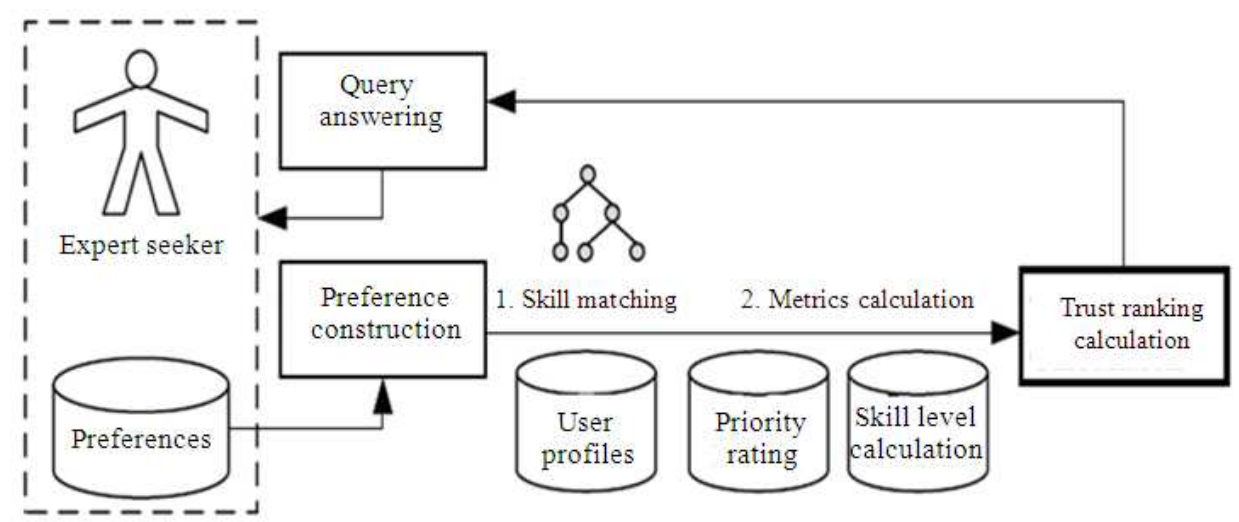

Fig. 2. Architecture for expert search 
Sridharan, K. and M. Chitra / Journal of Computer Science 10 (7): 1174-1185, 2014

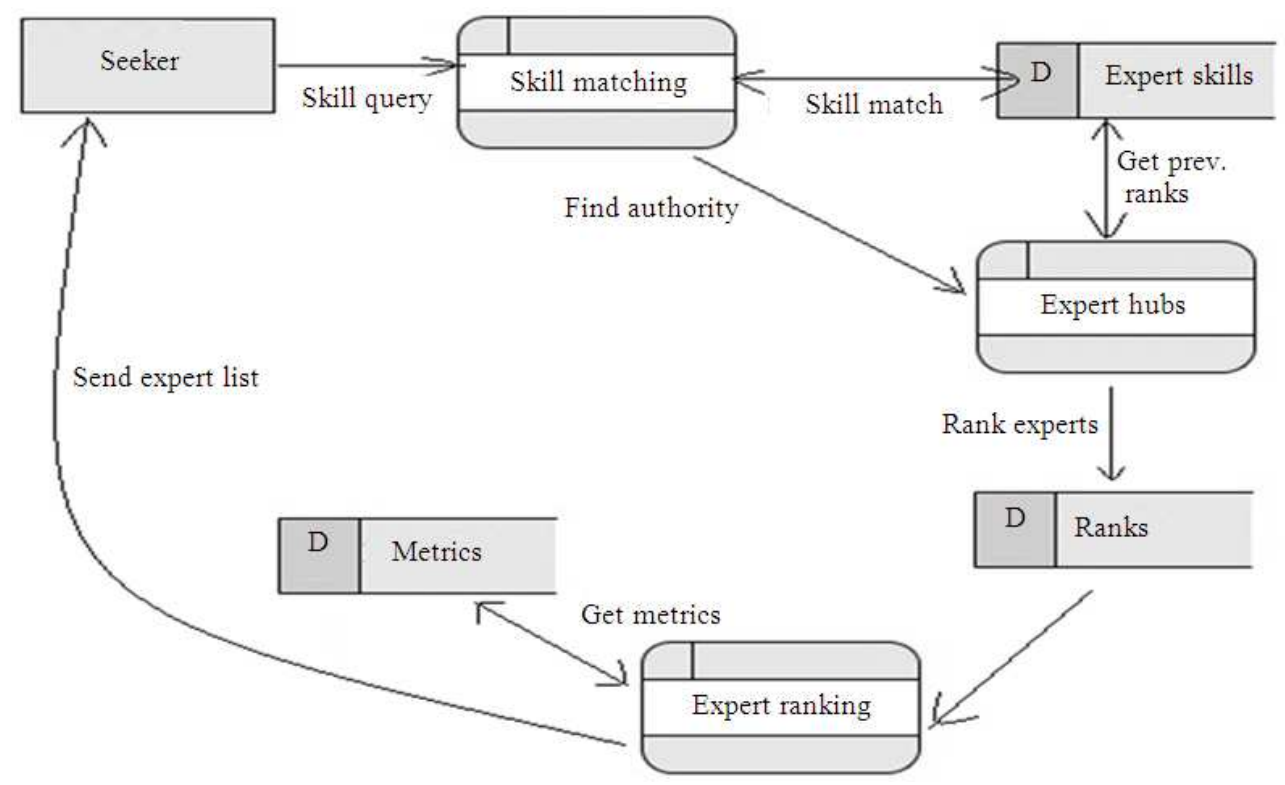

Fig. 3. Data flow diagram of expertise model

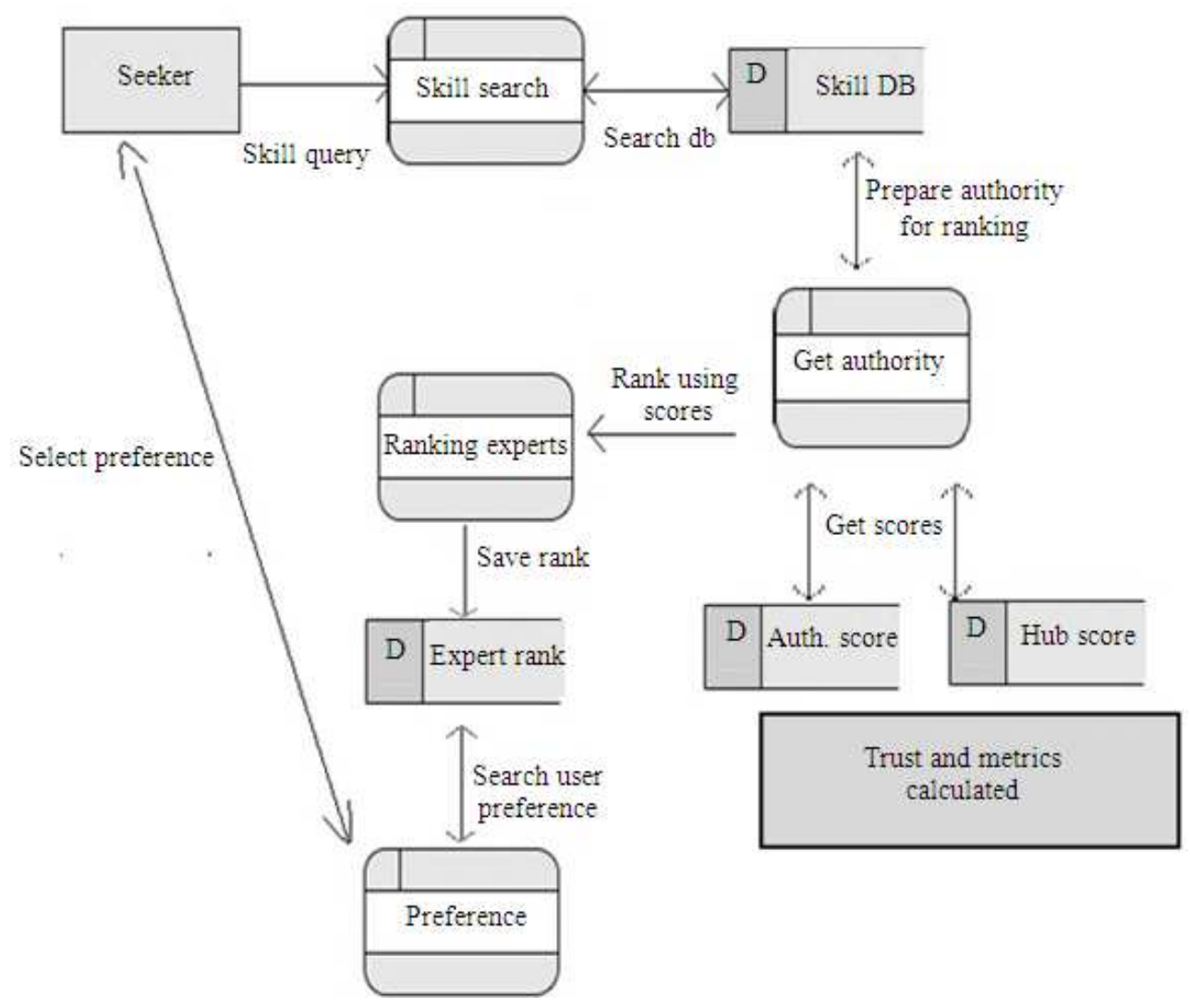

Fig. 4. Data flow diagram of expert discovery 
Expert hubs need to be exposed and this is influenced by social trust and rating mechanisms. Discovering hubs, Delegation actions when the expert fails to provide the answer, Trust based delegation patterns, rating procedure for rating experts, trust updates based on interactions from interaction metrics refer Fig. 3 and 4.

\subsubsection{Skill Matching Algorithm}

This present an algorithm supporting the concept of strong, weak and optional matching preferences through alternate approaches for calculating overlap similarities of sets of properties refer Fig. 2.

\subsubsection{Discovery of Experts}

The goal of our modified algorithm is to find proper, trustworthy and valid experts with respect to contextual information after verified and top rated.

\subsubsection{New Trust Algorithm}

The trust algorithm is processed right after the expert is being selected. This algorithm is to improve the trust of the query response seeker over the expert who is going to answer to the requested query.

\subsection{Delegation Model}

Delegation is the most important aspect of our system and requires a real time connection between expert seekers and the entire hub. When a seeker has found the right expert, he sends the RFS request for a problem. The receiving expert tries to solve the problem. If the expert cannot solve it, then he has the choice of rejecting the request or delegating it with other experts in the hub. In discovery of Experts, we present our expert discovery algorithm that is influenced by social trust and rating mechanisms. Its algorithm accounts for context information and weighted links between actors refer in Fig. 4. It shows how the expert is selected according to their skills.

Delegation is the most important aspect of our system and requires a real time connection between expert seekers and the entire hub. When a seeker has found the right expert, he sends the RFS request for a problem. The receiving expert tries to solve the problem. If the expert cannot solve it, then he has the choice of rejecting the request or delegating it with other experts in the hub.

If the receiving expert has not answered the query within a given a time limit then the query is converted to "failed to response" and the seeker is announced about its failure and he is requested to choose another expert.

In the existing system there is a use of triadic delegation pattern but here we do not use this pattern, this is done in order to overcome conflict over the response to the query.

In above related works and many other works similar to that of the related works there is a drawback and there is no proper query response search process, which can be very helpful for the seeker to get the precise or accurate response to the query and another drawback is; it is also a time consuming process for receiving such response.

In our proposed system, the 'Automatic Query formulation with Context Exploration and Recapture' will overcome the above drawback.We develop the layers for our proposed model and data flow for working schem is shown in Fig. 5 and 6 respectively. This can help the seekers to be more comfortable to view the desired document in a very short time, precise or accurate will be more and manual searching is reduced.

\begin{tabular}{|c|c|c|c|c|}
\hline \multicolumn{2}{|c|}{ Web browser } & Ranking & Dynamic server & Category \\
\hline User search & Professional search & \multicolumn{2}{|c|}{ Web server } \\
\hline \multicolumn{2}{|l|}{} & Expert system & Feedback system \\
\hline
\end{tabular}

Fig. 5. Layer diagram of AQFCER 


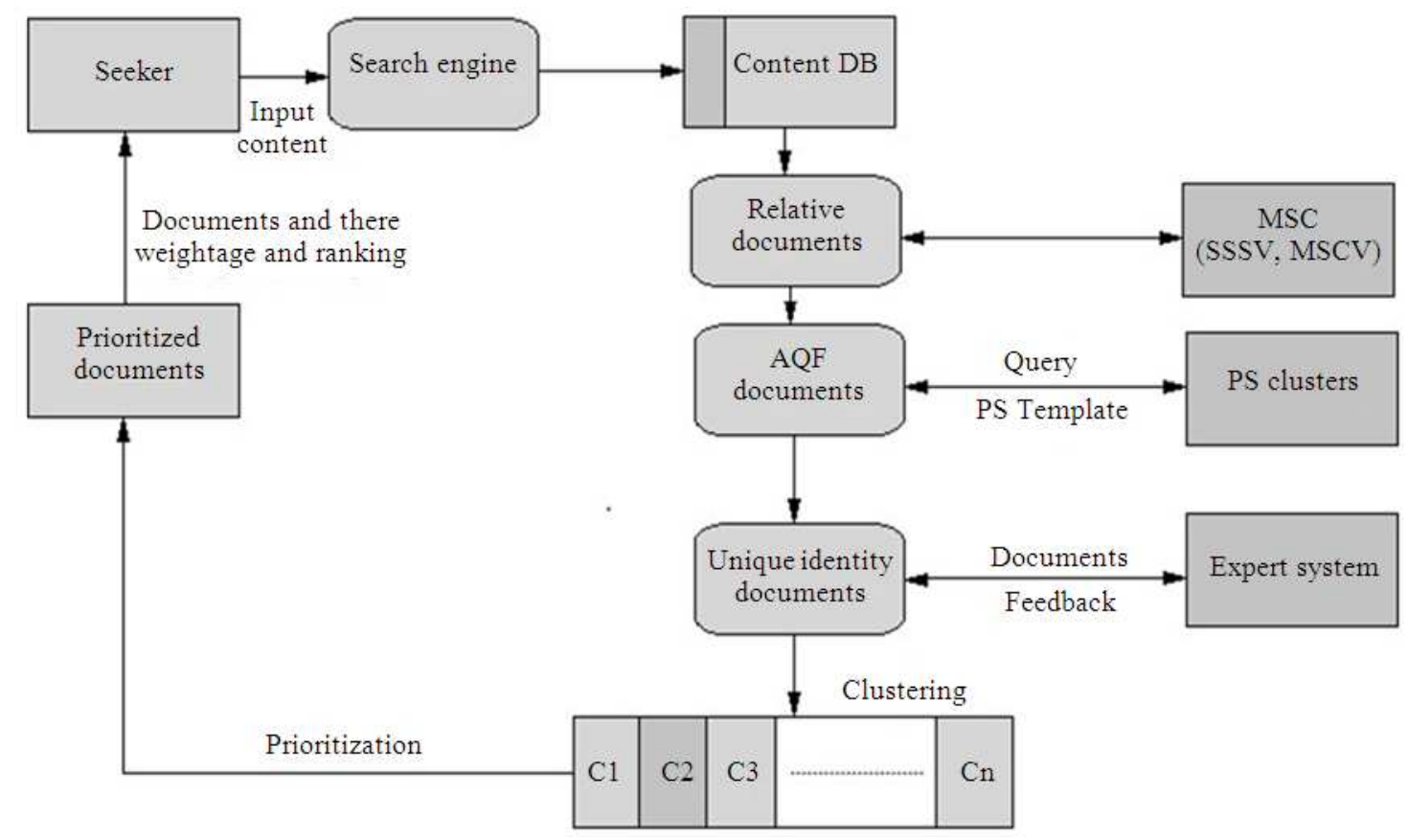

Fig. 6. Dataflow diagram of $A Q F$ with query context exploration and recapture

\section{AUTOMATIC QUERY FORMULATION WITH CONTEXT EXPLORATION AND RECAPTURE (AQFCER)}

Our proposed system refer in Fig. 1, provides a simple and special method; this search option is used to search the answer for the query provided by the seeker in a short span of time, with a maximum precision or accuracy and manual searching is reduced. It process the given query by search the relevant documents with single, two, three and more combination of keywords with less time. The relevant documents are forwarded to the next stage to get exact content from these documents by using automatic query formulation algorithm with help of prefix and suffix template based on the given query. This will be stored into corresponding dynamic cluster and refer in future with quick manner for the same keyword .It is very helpful for the seeker to get the answer quick and relevant with max precision and more accuracy. The execution of this system is as follows:

Firstly, there are 2 types of search:

- User search

- Professional search

\subsection{User Search}

This search is the ordinary search process which undergoes follows steps for execution: In Fig. 1, user search given query is consider and invoke the Multi Complex Search algorithm to get relevant docuemtns based on the combination of one or more keywords.After that we are applied the automatic query formulation algorithm to get exact relevant documents by possible prefix and suffis templates (PS template). Then the relevant doucments are forward to corresponding expert to make the more trust about its content quality to get score for it.Assign the document id by combination of key for verified documents and store into the corresponding cluster for retrieval. Rank the each documents again by each cluster to assign the proiroity. It will display into users based on the given query.

\subsubsection{Multi Complex Search Algorithm (MCS)}

It process the given query by search the relevant documents with single, two, three and more combination of keywords with less time. The Multi Complex Algorithm is processed as follows

\section{Algorithm:}

1. The set of keywords are combined to form a sentence 
2. The sentence is compared with the sentence available in the Relevant Documents

3. Each keyword is compared with the words in the documents

4. The formula for Single Simple Search can be written as:

If $($ Keyword $[\mathrm{k}]==$ documentword $[\mathrm{r}][\mathrm{k}])$

$$
\mathrm{SSSV}=\mathrm{SSSV}+1
$$

$>\mathrm{k}$ : Word in a line

$>$ r: Line number

> SSSV: Single Simple Search Value

5. If the value of SSSV is $=0$ then that document is not considered as relevant document

6. If the value of SSSV is $>0$ then that document is considered as relevant document

7. The set of keywords are combined to form a sentence.

8. The sentence is compared with the sentence available in the Relevant Documents.

9. The formula for the Multi Complex Search can be written as:

If (Sentence $[\mathrm{s}]==$ documentsentence $[\mathrm{r}][\mathrm{s}]$ )

$$
\begin{aligned}
\text { MCSV } & =\text { MCSV }+1 \\
& >\mathrm{s}: \text { a sentence } \\
& >\mathrm{r}: \text { line number } \\
& >\text { MCSV: Multi Complex Search Value }
\end{aligned}
$$

NOTE: Here a sentence is checked in such a way that the document gives' n' words equals the sentence to be searched and in that one word is removed from the back and a next new word is appended to the existing sentence.

10. If the value of MCSV is $=0$ then that document is not considered as original relevant document

11. If the value of MCSV is $>0$ then that document is considered as original relevant document

\subsubsection{Auto Query Formation(AQF) Algorithm}

The relevant documents are forwarded to the next stage to get exact content from these documents by using automatic query formulation algorithm with help of prefix and suffix template based on the given query shown in Fig. 6. The Auto Query Formation is processed as follows.

\section{Algorithm}

1. The query and the original relevant documents from the multi complex search algorithm are first taken as input on this process.
2. The structure of the query is viewed and the keywords are extracted

3. Based on the structure, the template is being constructed by adding the possible prefix and the suffix to those keywords, to form prefix template (pt) and suffix template (st).

4. The created templates are compared with the relevant document content and can be written as:

If $(\mathrm{pt}==$ documentsentence $[\mathrm{r}][\mathrm{t}])$

$\mathrm{AQFV}=\mathrm{AQFV}+1$

If $(\mathrm{st}==$ documentsentence $[\mathrm{r}][\mathrm{t}])$

$\mathrm{AQFV}=\mathrm{AQFV}+1$

$$
\begin{aligned}
& >\text { pt: prefix template } \\
& >\text { st: suffix template } \\
& >\text { r: line number } \\
& >\text { t: a sentence } \\
& >\text { AQFV: AQF value }
\end{aligned}
$$

5. If the value of $\mathrm{AQFV}$ is $=0$ then that document is not considered as the AQF Documents

6. If the value of AQFV is $>0$ then that document is considered as the AQF Documents

\subsubsection{Clustering}

These relevant documents are placed in the dynamic prioritized cluster. Methodology or functionality of clustering:

- The AQF Documents is provided with a unique identity number which is being found using the expert feedback details

- Those Documents are placed into the clusters C1, $\mathrm{C} 2 \ldots$ or $\mathrm{Cn}$ in their respective domain

\subsubsection{Prioritization}

The Documents are placed in the prioritized order with assigning ranks to it and it functions as follows:

- $\quad$ Feedback from Expert System (EFX)

- Content Based Ranking (CR)

- Auto Query Formation Rating (AQTV)

- Document View Count (DCV)

\section{Feedback from Expert System (EFX)}

After, every query is answered, for the first time the experts who are related to the respective domain will get the answered document. The Experts verify the answer and provides there feedback to the query. 
The Feedbacks received are viewed by the administrator and the unique identity number for the document is being provided, along with the document rating. Here we utilize the rating given by the experts (value range between $0-5$ points) for prioritizing the documents. The value received from this function is rounded off to 25 .

Mathematical formula:

$\mathrm{EFX}=\left(\sum \mathrm{FV} / \sum \mathrm{FE}\right) * 5$

$>$ EFX: Feedback for expert system value

$>\quad \sum F V$ : Sum of the experts feedback values

$>\quad \sum$ FE: Sum of experts

\section{Content Based Ranking (CR)}

It is used to identify the documents' weightage which is done using the algorithm given below:

\section{Algorithm}

1. Every time the source document is viewed for every document received from the domain clusters

2. Word comparison value is calculated

Mathematical formula:

$\mathrm{WC}=\left(\sum \mathrm{CW} / \sum \mathrm{TW}\right)^{*} 12.5$

$>$ WC: Word comparison value

$>\quad$ CW: Sum of the total no of words found equal, on comparison

$>$ STW: Sum of total no of words in document

Statement comparison value is calculated.

Mathematical formula:

$\mathrm{SC}=\left(\sum \mathrm{CS} / \sum \mathrm{TW}-\mathrm{CCW}\right) * 12.5$

$>$ SC: Statement comparison value.

$>$ ECS: Sum of total no of statement found equal, on comparison

$>$ LTW: Sum of total no of words in document

$>$ CCW: no of words in statement query

Content based ranking is calculated

Mathematical formula:

$\mathrm{CR}=\mathrm{WC}+\mathrm{SC}$

$>$ CR: Content based Ranking

$>$ WC: Word Comparison value

$>$ SC: Statement Comparison value

Science Publications
The CR value ranging between $0-25$ is being generated and returned.

The CR value is updated dynamically when every the document is viewed and updated and the CR is displayed.

\section{Auto Query Formation Rating (AQTV)}

This is the method which is used to get the value of the auto query formation template value and is calculated as Mathematical formula:

$$
\begin{aligned}
& \text { AQTV }=\left(\mathrm{AQSV} / \sum \mathrm{AQSV}\right) * 25 \\
&>\text { AQTV: Auto Query Formation Rating } \\
&>\text { AQSV: AQFV of the single AQF document } \\
&>\text { iAQSV: Sum of the AQFV of all AQF } \\
& \text { documents }
\end{aligned}
$$

\section{Document View Count (DCV)}

This is nothing but the number of times the document is viewed by the seekers, the value calculated ranges between $0-25$ and the generated value is returned.

Mathematical formula:

$\mathrm{DVC}=\left(\sum \mathrm{V} / \sum \mathrm{TV}\right) * 25$

$>\quad \sum \mathrm{V}$ : No of times the document is viewed

$>\quad \sum \mathrm{TV}$ : No of times the entire domain is visited

Combining the above 4 source values the priority value is provided to the documents which are displayed along with the documents in an orderly manner i.e. 'Original Prioritized Documents'.

The value of 'Prioritized Value' ranges between 0-100 and these values will be used to provide rank for the documents i.e., a rank provided based on prioritized value. This is calculated as follows:

$\mathrm{PV}=\mathrm{EFX}+\mathrm{CR}+\mathrm{AQTV}+\mathrm{DCV}$

$>$ PV: Prioritized Value

$>$ EFX: Feedback from Expert System

$>$ CR: Content Based Ranking

AQTV: Auto Query Formation Rating

$>$ DCV: Document View Count

Based on the Prioritized Value (PV) the document is ranked.

\subsection{Professional Search}

This search is an advanced search process, since the experts know what they are exactly searching for, so there is an option for the expert to search directly with 
providing set of details what they are exactly searching for.The expert query must be standard and describe exact requirement. In our proposed model refer Fig. 1. we are provide options for retrivel of exact documents with content accuracy. This direct searching will bring the search process move directly to the prioritized documents and from there he/she can browse through the documents faster.If the document is not found on 'professional search' then the expert undergoes the basic 'user search'one time only.

\section{RESULTS}

Google is one of the the most popular search engines on the internet for comparision and Google focusses on the link structure of web to determine the relevant results. Yahoo is the another well popular search engine to be consider for the comparition.

In google search engines, a challenge to compute the relevancy of Google for composite multi word queries $29.4 \%$ sites were fewer relevant, $28.6 \%$ of the sites were irrelevant followed by associations (20.4\%). It was also observed that $15.8 \%$ of the sites were more relevant and only a small percentage of sites (5.8\%) can't be accessed. The precision of Google for complex multiword queries was found to be 0.71 (Sampath Kumar and Prakash, 2009). After that it will increase 0.89 based on the improved algorithms proposed recently.

In our proposed search engine methods is comapre with this, it shows the precision is 0.93 and hence it is proved with sample data and graph.

For Yahoo, the search for composite multi-word queries outcomes showed that $34.6 \%$ of sites were a lesser amount of relevant while $26.8 \%$ of sites were unrelated. It was also experiential that 17.8 and $16.6 \%$ of sites were links and more pertinent respectively. The overall precision of the Yahoo was 0.76, after that it will increase 0.91 based on the improved algorithms proposed recently. In our proposed search engine methods is comapre with this, it shows the precision is 0.93 and hence it is proved with sample data and graph.

In our proposed search engine methods is comapre with this, it shows the recall value for google and yahoo is is 0.93 and hence it is proved with sample data and graph.

In Table 1. We are ploted the mean presision and recall value of google, yahoo and my proposed method AQF. The presicion for those search engines are calculated by total number of sites retrived, more relevant, less relevant, irrelevant, cant be accessed links for the given set of dfifferent queries.

The recall for those search engines are calculated by total number of sites retrived and sites relevant.

In Fig. 7 We proved the precision and recall value of our porposed method compare with famous search engines like google and yahoo.

We are consider top $\mathrm{n}$ documents for testing because the most of the relevant documents with more priority is listed within it for the given query .On execution of our proposed system we expect the output to be as follows:

- The document received by the seeker is accurate and precise

- The time take for receiving the exact document is less

- There is a less requirement of manual searching

- We have compared or test results with stack overflow and the yahoo ask search option

In Fig. 8 We proved the precision and recall value of our porposed method compare with famous search engines like Stackoverflow and yahooask.com.

In the Fig. 9 Time graph is drawn with respect to the time taken for the precise or accurate document to be received for the seeker at the last.

E.g., If there are 1000 documents and have to search one of them, let us now see step wise time taken for the document to be searched.This consume $0.15 \mathrm{sec}$ by MCS algorithm.

Table 1. Mean relative recall of Google, Yahoo and AQF

\begin{tabular}{llll}
\hline Search engines & Google & Yahoo & AQF \\
\hline Mean precision & 0.89 & 0.91 & 0.93 \\
Mean recall & 0.62 & 0.37 & 0.65 \\
\hline
\end{tabular}

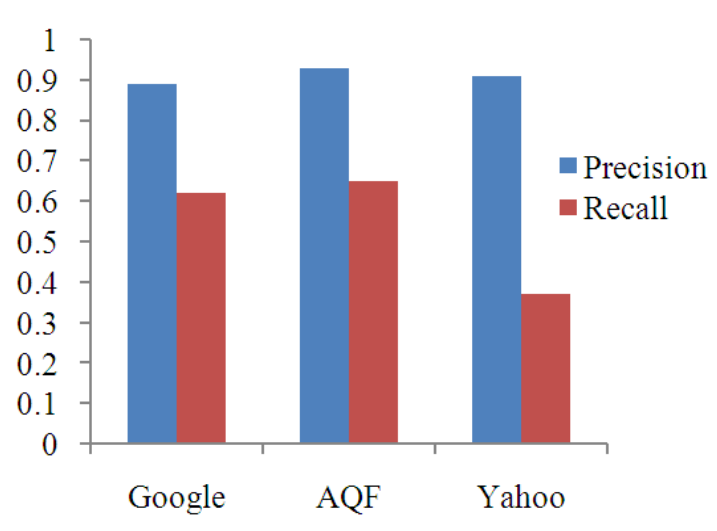

Fig. 7. The precision Vs. recall graph analysis 
Sridharan, K. and M. Chitra / Journal of Computer Science 10 (7): 1174-1185, 2014

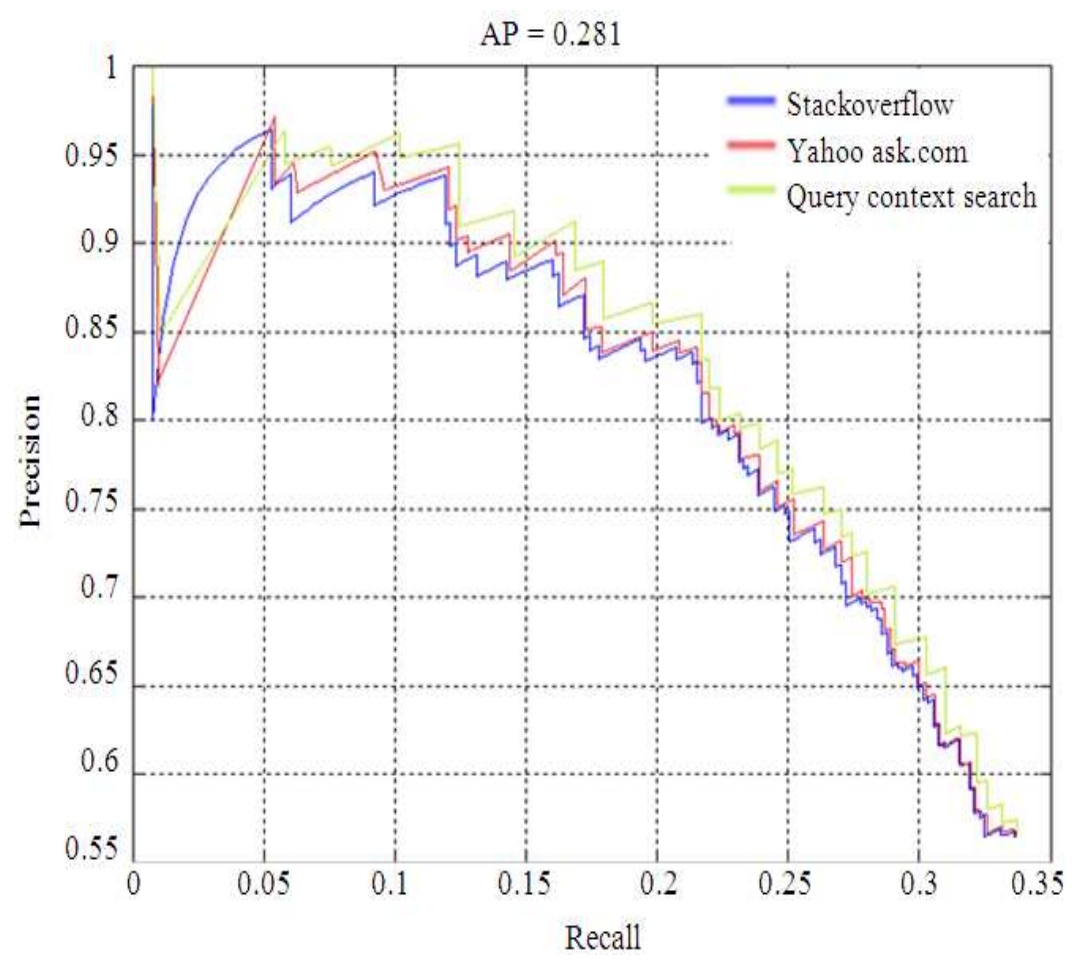

Fig. 8. The precision vs. recall graph analysis

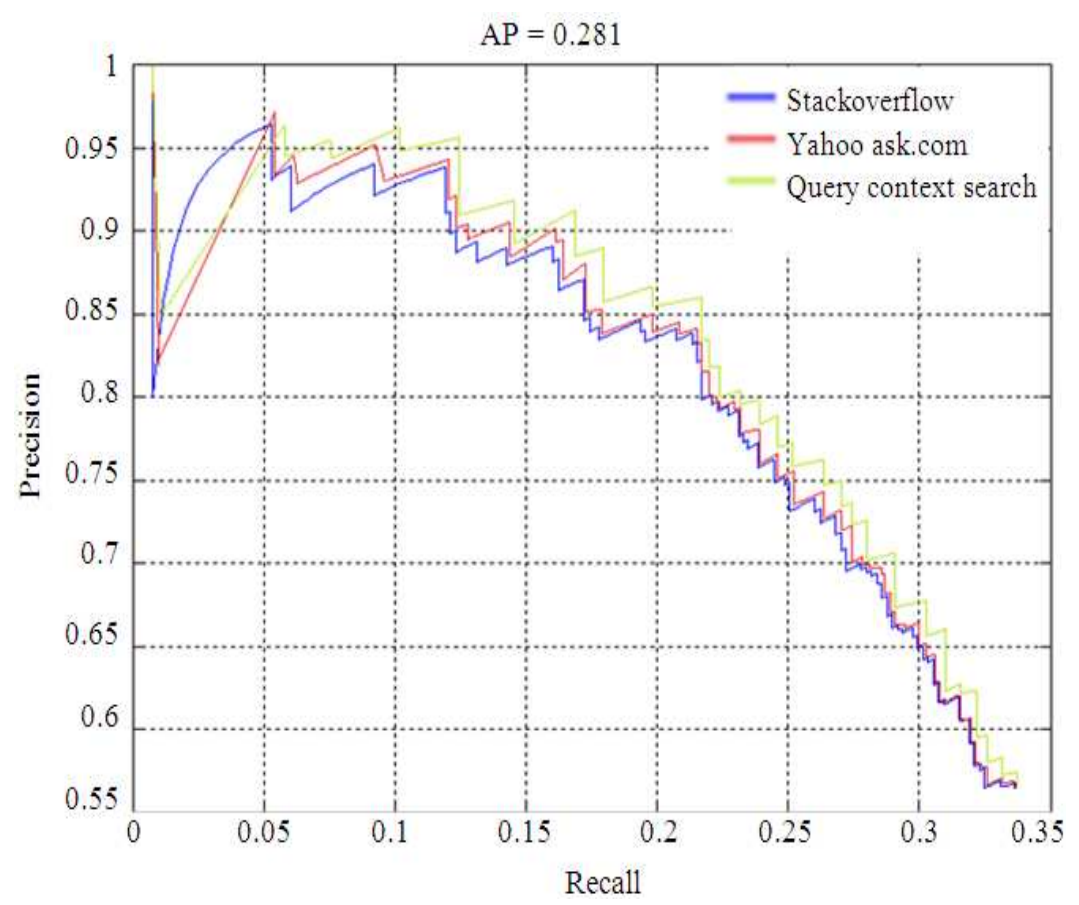

Fig. 9. The time Vs. no of documents graph analysis 


\section{DISCUSSION}

\subsection{Multi Complex Search (MCS) Algorithm}

- At this step the 1000 documents are checked for comparison with the query keywords

- 550 documents are found to match the keywords

- Once again the 550 documents are checked for comparison with the query keywords

- 250 documents are found to match the query keywords present

- This took $0.15 \mathrm{sec}$

\subsection{Auto Query Formation Template Algorithm}

- At this step the 250 documents are checked for comparison with the query keywords

- 50 documents are found to match the keywords present

- This took $0.15 \mathrm{sec}$

\subsection{Prioritized Documents}

- The 50 documents which are being produced after processing, the documents are ranked according to the calculated prioritized value.

- Since it has a processing of prioritizing and ranking it takes some extra time

- This took $0.1 \mathrm{sec}$

\subsection{Total Time Taken}

- $0.4 \mathrm{sec}$ for searching a document from 100 documents

Similarly for various other set of documents at various domains the document is searched.

\section{CONCLUSION}

We introduced the automatic query formulation with context exploration and recapture system using multi complex algorithm and automatic query formulation algorithm. It process the given query by search the relevant documents with single, two, three and more combination of keywords with less time. The relevant documents are forwarded to the next stage to get exact content from these documents by using automatic query formulation algorithm with help of prefix and suffix template based on the given query. It is very helpful for the seeker to get the answer quick and relevant with max precision and more accuracy. This proposed system of searching can help one step ahead in penetrating for response to the query with overcome the limitation of the existing method applied. The performance in improving precision and lowering recall is achieved through our system. It hence effectively reduces complexity in combining HPS with software services comparing with existing technique. Time, which is one of the most important scenarios, is considered here to be reduced on searching more. We are analyzing on the search engines features, which may further improve the trust and ranking of the search results with help of different feedback system. In future also it could be to investigate the performance/safety measures trade-off in better complexity.

\section{REFERENCES}

Agichtein, E., C. Castillo, D. Donato, A. Gionis and G. Mishne, 2008. Finding high-quality content in social media. Proceedings of the International Conference on Web Search and Data Mining, (WSDM '08), New York, pp: 183-194. DOI: 10.1145/1341531.1341557

Artz, D. and Y. Gil, 2007. A survey of trust in computer science and the semantic web. J. Web Semantics: Sci. Services Agents World Wide Web, 5: 58-71. DOI: 10.1016/j.websem.2007.03.002

Fryer, D., 2004. Federated search engines. Online, 28: 16-19.

Golbeck, J., 2008. Computing with Social Trust. 1st Edn., Springer, London, ISBN-10: 1848003560. pp: 348.

Golbeck, J., 2009. Trust and nuanced profile similarity in online social networks. J. ACM Trans. DOI: $10.1145 / 1594173.1594174$

Haveliwala, T.H., 2002. Topic-sensitive PageRank. Proceedings of the 11th International Conference on World Wide Web, May 07-11, New York, pp: 517526. DOI: $10.1145 / 511446.511513$

Jiang, C.B. and C. Li, 2010. Web log data preprocessing based on collaborative filtering. Proceedings of the Second International Workshop on Education Technology and Computer Science, Mar. 6-7, IEEE Xplore Press, Wuhan, pp: 118-121. DOI: 10.1109/ETCS.2010.588

Jurczyk, P. and E. Agichtein, 2007. Discovering authorities in question answer communities by using link analysis. Proceedings of the 6th ACM Conference on Conference on Information and Knowledge Management, Nov. 6-10, New York, pp: 919-922. DOI: $10.1145 / 1321440.1321575$

Kovac, D. and D. Trcek, 2009. Qualitative trust modeling in SOA. J. Syst. Architecture, 55: 255263. DOI: $10.1016 /$ j.sysarc.2009.01.002 
Liu, B. and K. Chang, 2004. Editorial: Special issue on web content mining. ACM SIGKDD Explorat. Newslett., 3: 1-4. DOI: 10.1145/1046456.1046457

Malik, Z. and A. Bouguettaya, 2009. Reputation bootstrapping for trust establishment among web services. IEEE Internet Comput., 13: 40-47. DOI: 10.1109/MIC.2009.17

Pazienza, M.T., 1997. Information Extraction: A Multidisciplinary Approach to an Emerging Information Technology. 1st Edn., Springer, Berlin, ISBN-10: 354063438X, pp: 213.

Peng, H., 2010. Discovery of interesting association rules based on web usage mining. Proceedings of the International Conference on Multimedia Communications, Aug. 7-8, IEEE Xplore Press, Hong Kong, pp: 272-275.DOI: 10.1109/MEDIACOM.2010.82

Ratnakumar, A.J., 2005. An implementation of web personalization using web mining techniques. J. Theoretical Applied Inf. Technol.

Rijsbergen, C.J.V., 1979. Information Retrieval. 2nd Edn., Butterworth.
Sampath Kumar, B.T. and J.N. Prakash, 2009. Precision and relative recall of search engines: A comparative study of Google and yahoo. Singapore J. Library Inform. Manage., 38: 124-137.

Schall, D., F. Skopik and S. Dustdar, 2011. Expert discovery and interactions in mixed service-oriented systems. IEEE Trans. Services Comput., 5: 233-245. DOI: $10.1109 /$ TSC.2011.2

White, R.W., S. Dumais and J. Teevan, 2009. Characterizing the influence of domain expertise on Web search behavior. Proceedings of the Second ACM International Conference on Web Search and Data Mining, Feb. 9-11, New York, pp: 132-144. DOI: $10.1145 / 1498759.1498819$

Yang, J., L.A. Adamic and M.S. Ackerman, 2008. Competing to share expertise: The tasken knowledge sharing community. University of Michigan.

Witten, E., 1994. Monopoles and Four-Manifolds. Math.Res.Lett.1: 769-796.

Kosala, R. and H. Blockeel, 2000. Web mining research: a survey. ACM SIGKDD Explorat. Newslett., 2: 15. DOI: $10.1145 / 360402.360406$ 